
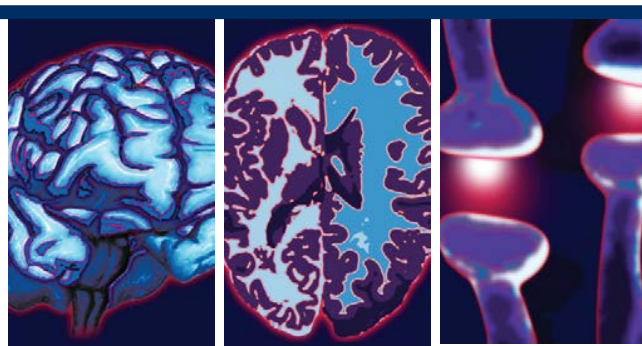

\title{
Potential Benefits of Melatonergic Treatment in Schizophrenia: Ameliorating Demyelination via Inhibiting CDK5-Mediated Hyper-Autophagy
}

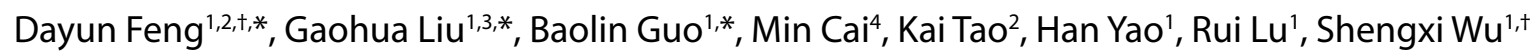

\begin{abstract}
Objective

Impairment of melatonergic activity, including alteration of melatonin secretion and melatonin receptor (MT) modifications, has been linked to the pathophysiology of schizophrenia. However, its definite therapeutic effect on schizophrenia-like symptoms and the biological mechanism are still illusive. The present study aimed to explore the potential benefis of melatonergic treatment in schizophrenia and underlying molecular mechanism.
\end{abstract}

\section{Methods}

A cuprizone (CPZ) -induced schizophrenic mice model was established. Agomelatine, a MT1 and MT2 agonist, was administrated intraperitoneally. Open field, three-chamber and Y-maze tests were performed for evaluation of behavioral and cognitive deficits. The myelination was examined in corpus striatum of mice. In vitro, alterations of cyclin-dependent kinase 5 (CDK5) expression, autophagic flux and apoptotic index were detected in OLN93 oligodendrocytes.

\section{Results}

We demonstrate that treatment with agomelatine exerts a significant improvement of the behavioral and cognitive deficits in CPZ-induced schizophrenic mice. Agomelatine also suppresses the excessive autophagy and rescues the myelination in corpus striatum in CPZtreated mice. Notably, all these effects of agomelatine are markedly weakened by an autophagy inducer Rapamycin (RAPA). In vitro, cuprizone dose- and time-dependently induces excessive autophagy and autophagic flux, therefore depresses cell viability in oligodendrocytes. Interestingly, CDK5 exerts positive regulation in CPZ-induced autophagy, revealing that CDK5 overexpression enhances LC3 II level and autophagosome formation in company with cuprizone treatment, while CDK5 knockdown works conversely. Importantly, agomelatine suppresses CDK5 expression and autophagic flux against cuprizone, which to the contrary is blocked by CDK5. Meanwhile, agomelatine also inhibits stress-associated activation of p38

\footnotetext{
'Department of Neurobiology School of Basic Medicine, Fourth Military Medical University, Xi'an 710032, P.R. China

2Department of Neurosurgery and Institute for Functional Brain Disorders, Tangdu Hospital, Fourth Military Medical University, Xi'an 710038, P.R. China

${ }^{3}$ Department of Psychiatry, General Hospital of Air Force, Beijing 100142, P.R. China

${ }^{4}$ Department of Psychiatry, Xijing Hospital, Fourth Military Medical University, Xi'an, 710032, P.R. China

${ }^{\dagger}$ Author for correspondence: Shengxi Wu, Department of Neurobiology, School of Basic Medicine, Fourth Military Medical University, Xi'an 710032, P.R. China, (Tel) +86-29-84774557; email: shengxi@fmmu.edu.cn

Dayun Feng, Department of Neurobiology, School of Basic Medicine; and Department of Neurosurgery, Tangdu Hospital, Fourth Military Medical University, Xi'an 710038, P.R. China, (Tel/Fax) +86-29-84717556; email: dayunfmmu@163.com

*Authors contributed equally to this project.
} 
and c-Jun- $\mathrm{N}$-terminal Kinase (JNK) induced by cuprizone in CDK5-dependent manner, as well as reducing cellular apoptosis.

\section{Conclusion}

The present findings, for the first time, link autophagy-demyelination in schizophrenia, and strongly suggest that melatinergic treatment possesses potential benefits against schizophrenia through ameliorating demyelination by inhibiting CDK5-mediated autophagy.

\section{Keywords}

Schizophrenia; Autophagy; Autophagic flux; Agomelatine; CDK5; Demyelination; Oligodendrocyte.

\section{Introduction}

Schizophrenia is one of the most detrimental mental illnesses characterized by psychosis, apathy, social withdrawal, and cognitive impairment, and affects about $1 \%$ of the population worldwide [1]. White matter loss, secondary to oligodendrocyte death and demyelination, is characterized in schizophrenia, as well as other demyelinating diseases like multiple sclerosis and depression [2].

Presently, the oligodendrocytic pathology is demonstrated to be one of the key components of schizophrenia. Reductions of neuropil, and myelin [3], altogether with decreased numbers of oligodendrocytes [4] were found in schizophrenia. Microarray analysis studies implied that the oligodendrocyte-specific genes were decreased in the prefrontal cortex of schizophrenia patients compared with brain tissues from the Control group [5-7]. Cuprizone (CPZ), a copper chelator that causes consistent demyelination and mature oligodendrocyte loss in the central nervous system (CNS), is extensively adopted to study demyelination and remyelination [8], and to model demyelination and schizophrenia like behaviors in rodents [9]. However, little is known about the process of white matter losing and the underlying molecular mechanism.

Melatonergic system has been involved in the pathophysiology of schizophrenia since the first decade of the twentieth century. The smaller volumetric changes of the pineal gland were observed in patients with schizophrenia by radiological evaluation [10]. The hypocrine of pineal leading to decreased melatonin secretion was also indicated to be related in the psychosis and behavioral deficits [11,12], which was also mentioned as a biological marker of schizophrenia [13].

Moreover, there is a noteworthy implication between the promoter polymorphism of the MT gene and schizophrenia [14,15], especially MT1A may be a susceptibility gene for schizophrenia. Agomelatine is a novel antidepressant that acts as an agonist for melatonin MT1 and MT2 receptors, as well as an antagonist on serotonin 5HT2C receptor [16]. It is effective in treating depression, bipolar disorder, anxiety disorder, alcohol dependence, migraine, schizophrenia, etc [16]. Even though small-scale of clinical cases reports and experimental investigations suggested melatonin or MT agonist Agomelatine possessed potential for improving schizophrenia-like symptoms $[11,17,18]$, the definite therapeutic effect on schizophrenia-like symptoms and the biological mechanism are still illusive.

Autophagy is a lysosome-dependent degradation pathway that controls intracellular turnover of cytoplasmic components and organelles, which is essential for cell survival whose abnormality leads to activation of apoptosis [19]. Recent studies showed that disturbed autophagy played a key role in the pathophysiological process of multiple psychiatric disorders [20-22]. Further evidences indicated restoration of autophagy function ameliorated schizophrenia-like behaviors in an autophagy deficient mice model [23]. CDK5 is a key regulator of cell-cycle progression, which has been demonstrated to play critical role in stress induced excessive autophagy associating with cell survival recently $[24,25]$. Besides, increased CDK5 signaling in the nucleus accumbens (NA) in a schizophrenia model and downregulation of CDK5 by antipsychotics in schizophrenia patients has been reported [26]. Despite all this, whether autophagy and CDK5 are involved in the damage of oligodendrocyte is elusive.

The present study aimed to examine the potential benefis of melatonergic treatment in a CPZinduced schizophrenia model on the behavioral changes and damaged white matter. And we 
further investigated the effects of cuprione and agomelatine on the process of autophagic flux and apoptosis in OLN93 oligodendrocytes. We also detected the role of CDK5 as a possible modulator in CPZ-mediated hyper-autophagy and agomelatine-mediated neuroprotection in oligodendrocytes. The findings will improve our understanding the pathological mechanism and the application of melatonergic treatment in schizophrenia.

\section{Methods \\ - Animal preparation and experimental protocols}

The study was approved by the Committee of Animal Care and Use for Research and Education (CACURE) of the Fourth Military Medical University (Xi'an, China), and complied with the National Institutes of Health (NIH) guidelines for the care and use of laboratory animals.

Eighty-four adult male C57BL/6 mice (8-10 week-old, $25.0 \pm 5.0 \mathrm{~g}$ ) were used in this study, and maintained on a 12: $12 \mathrm{~h}$ light: dark cycle under an ambient temperature of $22-25^{\circ} \mathrm{C}$ with food and water available ad libitum. Mice were divided into five groups: Control group, CPZtreated group, AGT-treated group, CPZ+AGTtreated group and $\mathrm{CPZ}+\mathrm{AGT}+\mathrm{RAPA}$ group. The schizophrenia model was established according to our previous report [27], feeding with rodent chow mixed with cuprizone at $0.2 \% \mathrm{w} / \mathrm{w}$ consecutively for 5 weeks. The Control group was received normal chow accordingly. Agomelatine (AGT, $50 \mathrm{mg} / \mathrm{kg} /$ day, Selleck Chemicals, TX, USA) was administrated intraperitoneally (i.p.) for the final 4 weeks at 18:00 daily. Rapamycin (RAPA, $4 \mathrm{mg} / \mathrm{kg} / \mathrm{day}$, Selleck Chemicals, TX, USA) was administrated intraperitoneally at 16:00 daily along with the agomelatine injection period in the $\mathrm{CPZ}+\mathrm{AGT}+\mathrm{RAPA}$ group. In the last three days of the intervention period, all mice were subjected to open field, Y maze and three-chamber test. On day 36, all mice were sacrificed under anesthesia and their brains were removed for Western blotting and immunofluorescent staining.

\section{- Behavioral test}

Open field, three-chamber and Y-maze tests were performed as described previously [28]. Open field test was adopted to measure the locomotion and anxiety levels as rodents tend to move close to the walls, and at the same time have the characteristic to test individually when separated from the social group. In brief, a mouse was initially placed in the center of the apparatus $(25 \mathrm{~cm} \times 25 \mathrm{~cm} \times 45 \mathrm{~cm}, 340 \mathrm{lux})$, and a video camera was placed above it to monitor the animals' movements. During a 5 min period, the bouts, time and distance spent in the central area were analyzed to detect the anxiety level.

The three-chamber test was used to evaluate social affiliation and social preference, which could mimic social withdrawal, a key component of the negative symptoms of schizophrenia. The experimental apparatus was comprised of a rectangular, three-chamber box. Each chamber was $30 \mathrm{~cm} \times 30 \mathrm{~cm}$ and divided by two Plexiglas boards. Two wire containment cups with removable lids that large enough to hold a single mouse were fixed in the middle of each side chamber. Before the experiment began, mice were put into the behavioral room for 30 min. In the adaptation session, the right and left chambers were isolated with the Plexiglas boards, and the subject mouse was placed at the center of the middle chamber to habituate for $5 \mathrm{~min}$. In session 1, one of the control mice (stranger 1) was put inside a wire containment cup and located in one of the side chambers. The Plexiglas boards were removed to allow the subject to freely explore the three chambers. In session 2 , a second control mouse (stranger 2) was placed inside an identical wire containment cup in the opposite chamber that had been empty during session 1 . The duration of each session was $10 \mathrm{~min}$ and monitored with a video camera. The sociability was evaluated by analyzing the bouts, duration and distance of the subject mouse in each chamber.

Y-maze test was used in the present study to evaluate the spatial working memory of cuprizone and AGT treated mice. The apparatus was comprised of three arms $(30 \mathrm{~cm} \times 10 \mathrm{~cm} \times$ $25 \mathrm{~cm}$, positioned $45 \mathrm{~cm}$ high from the ground) positioned at an angle of $120^{\circ}$ and surrounded by various extra-maze cues. Each mouse was positioned in the center of the maze and let it to explore for $8 \mathrm{~min}$. The order of the exploration of each arm was recorded manually. The actual alternation was defined as entries into all three arms on consecutive occasions. Therefore, the maximum alternation was the total number of arm entries minus two, and the percentage of alternation was calculated as (actual alternations / maximum alternations) $\times 100$.

\section{- Cell culture, virus infection, and drug administration}

OLN93 cell was a gift generously from Doc. Zhao Xianghui (Fourth Military Medical 
University). Cells were planted at a density of $1 \times 10^{5} / \mathrm{cm}^{2}$ in $60 \mathrm{~mm}$ Petri dishes (Nunc, MA, USA) and routinely cultured in DMEM high glucose supplemented with $10 \%$ FBS (Gibco, CA, USA) and $1 \%$ penicillin-streptomycin mixture (Sigma, St. Louis, MO, USA) in a humidified incubator $\left(37^{\circ} \mathrm{C}, 5 \% \mathrm{CO}_{2}\right)$.

To evaluate autophagic flux, cells were transfected with mRFP-GFP-LC3 adenovirus (50 MOIs, Hanbio Biotechlogy Co., Ltd., Shanghai, China) according to the manufacturer's instructions for $24 \mathrm{~h}$. For cell stimulation and drug intervention, cuprizone (Sigma, St. Louis, MO, USA) and agomelatine were dissolved in $10 \%$ dimethyl sulfoxide (DMSO) and added to the OLN93 cells at various concentrations $(5,10,20 \mu \mathrm{M})$ for different time $(6,12,24 \mathrm{~h})$.

\section{- Western blot analysis}

Protein lysates were prepared from ventrolateral striatum of mice and OLN93 cell cultures using RIPA buffer (containing $20 \mathrm{mM}$ Tris, 150 mM NaCl, $0.1 \%$ SDS, $1 \%$ NP40) mixed adequately with a 100:1 (v/v) ratio of protease and phosphatase inhibitor (Roche, Basel, Switzerland). Total $20 \mu \mathrm{g}$ samples quantified using BCA Protein Assay Kit (Thermo, Rockford, IL, USA) were separated by sodium dodecyl sulfate-polyacrylamide gels (SDS-PAGE) and transferred to PVDF membranes (Roche, Basel, Switzerland) using the Mini-PROTEIN electrophoresis system (Bio-Rad, California, USA). After being blocked for $1 \mathrm{~h}$ in $5 \%$ non-fat milk, membranes were incubated overnight with the primary antibodies at $4{ }^{\circ} \mathrm{C}$ with following antibodies: anti-LC3 (1:1000, Cell Signaling Technology), anti-CDK5 (1:2000, Cell Signaling Technology), anti-phospho-p38 (1:1000, Cell Signaling Technology), anti-p38 (1:2000, Cell Signaling Technology), anti-p-JNK (1:1000, Cell Signaling Technology), anti-JNK (1:1000, Cell Signaling Technology), and anti-MBP (1:2000, Cell Signaling Technology). After incubation with HRP-conjugated secondary antibodies the secondary antibodies, blots were detected with an ECL system (Zetalife, Menlo, CA, USA). The signals were visualized on X-ray films and analyzed with Image J NIH software (version 1.48).

\section{- Immunofluorescence staining}

Animals were anesthetized with pentobarbitaland perfused with $50 \mathrm{ml} 0.01 \mathrm{M}$ PBS (pH 7.4), followed by $50 \mathrm{ml} 4 \%$ paraformaldehyde in phosphate buffer ( $\mathrm{pH}$ 7.4). After being cryoprotected for $24 \mathrm{~h}$ in $30 \%$ sucrose, coronal brain sections $(25 \mu \mathrm{m})$ of prefrontal cortex $(0.5-$ $1.1 \mathrm{~mm}$ from bregma) were collected with a cryotome. Sections were incubated with antiMBP antibody in dilution buffer (0.01 M PBS containing $2 \%$ goat serum and $0.3 \%$ Triton X-100). Alexa Fluor 488/594-conjugated IgG (1:500; Invitrogen, Eugene, OR, USA) was used for immunofluorescence labeling. As a distinct demyelination was identified in the ventrolateral striatum in CPZ-treated mice according to previous reports $[29,30]$, images in this region were captured with a laser confocal microscopy (Olympus, FV1200, Tokyo, Japan).

\section{- Autophagic flux determination}

After adenovirus transfection and drug admistration for $24 \mathrm{~h}$, the cells cultured in laser confocal dishes (at a density of $1 \times 10^{4} / \mathrm{cm}^{2}$ ) fixed with $4 \%$ paraformaldehyde. Images were captured with the laser confocal microscopy. The autophagic flux analysis was performed according to our previous study [31]. As GFP degrades in acidic environment while RFP does not, the yellow spots (formed out of the overlap between red and green) indicate autophagosomes, while the red spots indicate autophagic lysosomes. The increase of the ratio in red and yellow spots fluorescence intensity indicates the enhancement of autophagic flux.

\section{- Cell viability and TUNEL assays}

Cells were planted in laser confocal culture dishes (NEST Biotechnology, Wuxi, China) for MTT assay and in 96-well plates (Nunc, MA, USA) for TUNEL assay according to our previous report [32]. Cell viability was determined by the conventional 3-(4,5-dimethylthiazol-2yl)2,5-diphenyltetrazolium bromide (MTT) assay (Millipore, Merck, Darmstadt, Germany). The absorbance was read at $570 \mathrm{~nm}$ using a microplate reader (Bio-Rad, Hercules, CA, USA). The TdT-mediated dUTP nick end labelling (TUNEL) assay was performed using a TUNEL Apoptosis Kit (Calbiochem, Merck, Darmstadt, Germany). The fluorescence was captured with the laser confocal microscopy. All experiments were performed in accordance with the manufacturers' instructions.

\section{- Statistical analysis}

The data were analyzed with the SPSS 19.0 software, and are expressed as mean \pm standard error of mean (SEM). The results were analyzed by one-way analysis of variance (ANOVA), followed by the Newman-Keuls multiple 
comparison test. Statistical significances were considered as a value of $\mathrm{P}<0.05$.

\section{Results}

- Targeted MT treatment improved the schizophrenia-like symptoms through mediating autophagy in CPZ-treated mice

The effects of agomelatine treatment on the schizophrenia-like symptoms, as well as the potential association with autophagy, were examined by open field, three-chamber and Y-maze tests. In the open field test Figure 1A,B, compared with the control mice, treatment of agomelatine alone slightly increased the distance traveled in the central area. Cuprizone significantly decreased the duration $P<0.05$, Figure $1 \mathrm{~A}$ and distance $P<0.05$, Figure $1 \mathrm{~B}$ in the central area, which implied a manifestation of anxiety-like behaviors. In contrast, the two parameters were reversed by agomelatine treatment in the CPZ + AGT group $(P<0.05$, compared with the $\mathrm{CPZ}$ group, respectively), indicating that agomelatine exerted anxiolytic effect. However, the improving effect of agomelatine was largely blocked by additional autophagy induction with RAPA $(P<0.05$, the $\mathrm{CPZ}+\mathrm{AGT}$ group compared with the CPZ + AGT + RAPA group, respectively).

Social interaction impairment was a correlate of social withdrawal, a key component of the negative symptoms of schizophrenia. In the CPZ group, mice showed impaired social affiliation and social preference in the three chamber test Figure 1C,D, which were restored by agomelatine co-administration. Specifically, $\mathrm{CPZ}$ group mice spent less time in the chamber holding Stranger 1 during session $1 \quad(P<0.05$,
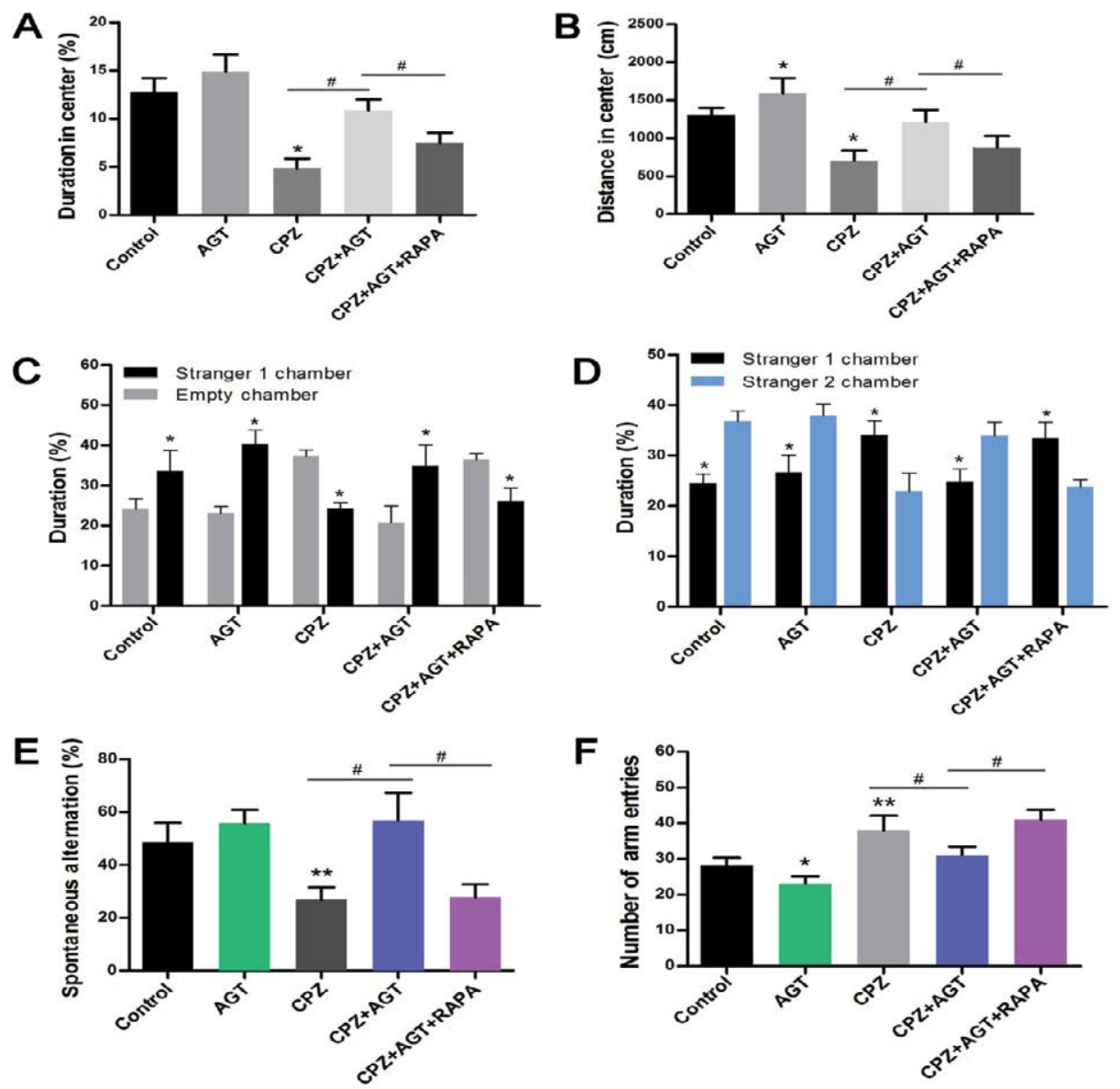

$\mathbf{F}$

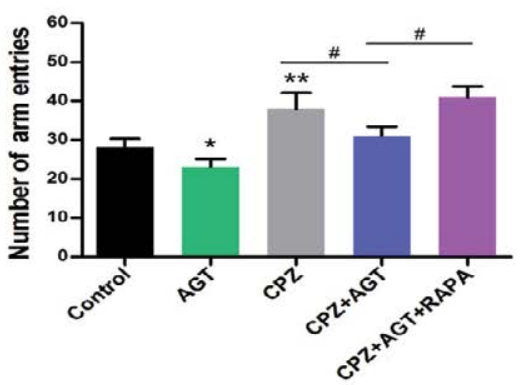

Figure 1: The effects of agomelatine and rapamycin on schizophrenia-like symptoms in CPZ-treated mice.

Control and experimentally treated mice were subjected to open field, three-chamber and $\mathrm{Y}$-maze tests.

$A, B$. The duration (A) and distance (B) that the mice traveled in the central area in open field test.

C,D. The duration spent in the empty chamber or Stranger 1 chamber during session 1 (C) and the duration spent in the Stranger 1 chamber or Stranger 2 chamber during session 2 (D) in three-chamber test. E,F. The spontaneous alternation $(E)$ and the number of arm entries (F) of mice in $Y$-maze test. Data are expressed as mean \pm SEM $\left(n=12-16\right.$ mice per group). ${ }^{*} P<0.05,{ }^{* * *} P<0.01,{ }^{*} P<0.05$. 
compared with the Control group, Figure 1C, and spent less time with Stranger 2 during session $2(P<0.05$, compared with the Control group, Figure 1D. After treatment with agomelatine, the social behaviors were restored in the CPZ + AGT group that mice spent more time with its conspecifics than the empty cage $P<0.05$, Figure 1C, and a novel other than a familiarized one (Stranger 1) $P<0.05$, Figure 1D. Notably, extra treatment with RAPA markedly weakened the above effect of agomelatine $(P<0.05$, compared with the CPZ + AGT + RAPA group, respectively).

In the Y-maze tests (Figure 1E,F), Agomelatine alone decreased the number of arm entries compared to the controls $(P<0.05$, Figure 1F). Mice in the CPZ group showed decreased spontaneous alternation $(P<0.05$, Figure $1 \mathrm{E})$ and increased total arm entries $(P<0.05$, Figure 1F) compared with the Control group. In addition, significant reversals of these alteration induced by CPZ-exposure were found in the $\mathrm{CPZ}+\mathrm{AGT}$ group $(P<0.05$, Figure 1E,F). All these effects of AGT were blocked by additional treatment with RAPA $(P<0.05$, Figure 1E,F).

Our results suggested that agomelatine exerted a significant improvement of the behavioral deficits in CPZ-induced schizophrenia mice, and implied a potential mechanism through mediating autophagy.

\section{- Excessive autophagy was associated with the antidemyelination of agomelatine in schizophrenia mice}

We further investigated the effect of agomelatine on demyelination and the alteration of autophagy in CPZ-treated mice. Brain tissues from the ventrolateral striatum were collected for Western blotting as a distinct demyelination was identified in this region according to previous reports. As was shown in Figure 2A, cuprizone enhanced the conversion of LC3- I into LC3II and decreased the MBP expression $(P<0.01$, compared with the Control group). Lower LC3II level was found in the AGT group but was not significant. Agomelatine co-administration in CPZ-exposure mice significantly inhibited LC3-II formation and increased the MBP expression $(P<0.05$, compared with the $\mathrm{CPZ}$ group). The effects of agomelatine on LC3 and MBP were completely blocked by additional RAPA treatment $(P<0.05$, compared with the $\mathrm{CPZ}+\mathrm{AGT}+\mathrm{RAPA}$ group). The demyelination in CPZ-treated mice was also detected by immunofluorescence staining of MBP. As was shown in Figure 2B, severe demyelination was observed in the ventrolateral striatum compared with the Control mice. Agomelatine treatment showed a notable mitigation of the demyelination induced by cuprizone, while which was weakened by RAPA. The result of immunofluorescence density analysis was similar to the blots above. These findings suggested that agomelatine could rescue the myelination in schizophrenia mice might through suppressing the excessive autophagy induced by cuprizone.

\section{- Cuprizone suppressed cell viability and enhanced autophagy flux in oligodendrocytes}

For further investigating the mechanism of demyelination, we examined the cell viability and autophagy process in cuprizone-treated OLN93 oligodendrocytes. As was shown in Figure 3A, using MTT assay, we found that cuprizone doseand time- dependently induced death of OLN93 cells. Notably, $10 \mu \mathrm{M}$ of cuprizone treatment for $24 \mathrm{~h}$ resulted in about $50 \%$ of cell death. Moreover, cuprizone significantly enhanced the conversion of LC3- I into LC3- II in dose(Figure 3B) and time- (Figure 3C) dependent manner $(P<0.05$, compared with the Control group), respectively. Thus, $10 \mu \mathrm{M}$ of cuprizone for $24 \mathrm{~h}$ treatment was chosen in the following treatment.

For autophagic flux assay, different dosages of CPZ treatment for $24 \mathrm{~h}$ were performed following transfection of mRFP-GFP-LC3 for $24 \mathrm{~h}$. The results showed CPZ dose-dependently increased the number of mRFP-LC3 dots representing autophagolysosomes in the merged images in OLN93 cells, and also decreased the yellow dots representing autophagosomes accordingly, suggesting that the autophagic flux in OLN93 cells was enhanced by CPZ treatment (Figure 3D). Importantly, $20 \mu \mathrm{M}$ of CPZ induced excessive reductions of mRFPLC3 dots and yellow dots, as well as obviously vacuolar degeneration in OLN93 cells. The flux rate of autophagy (Figure 3E) was evaluated by the ratio of autophagolysosomes (red dots in the merged phases) and autophagosomes (yellow dots in the merged phases). We found that the autophagolysosomes were increased, while the autophagosomes were reduced after different dosages of cuprizone treatment for $24 \mathrm{~h}(P<0.01$, $P<0.001$, compared with the Control group).

- CDK5 was positively involved in CPZinduced autophagy in oligodendrocytes

Strong association of CDK5 and schizophrenia 

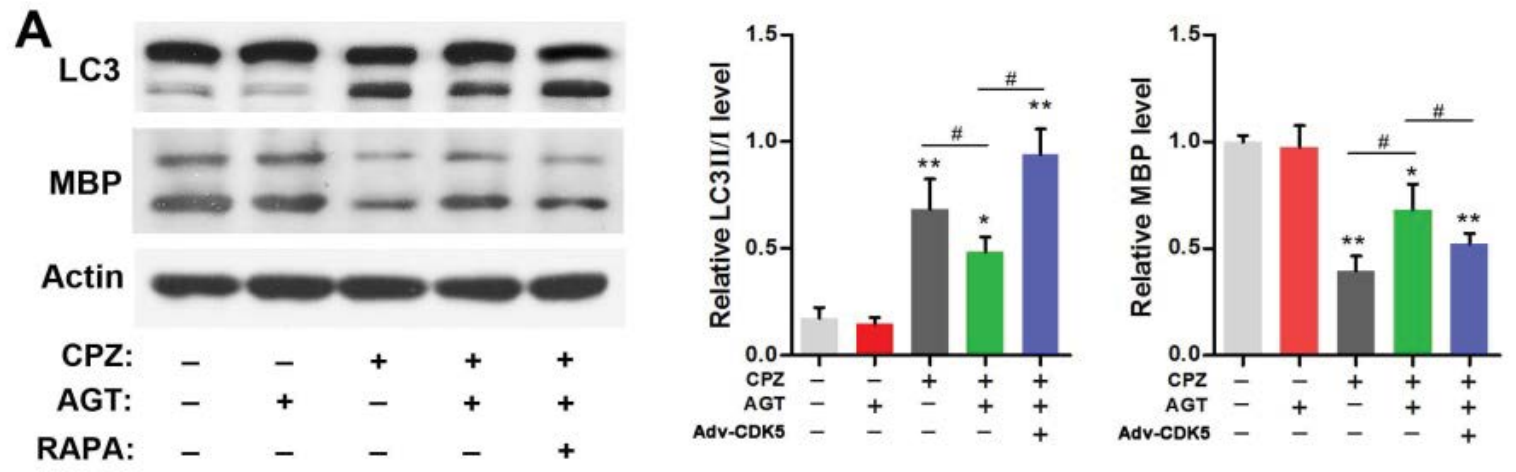

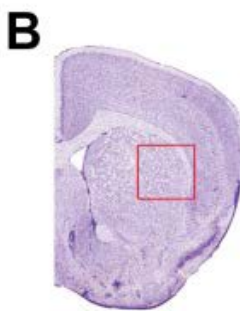

CPZ

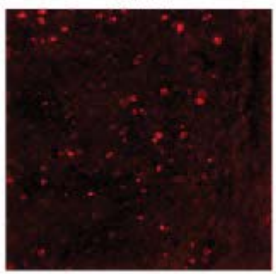

Control

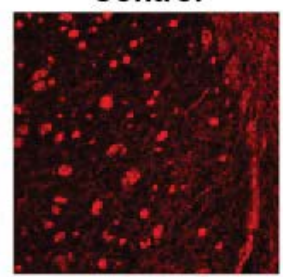

CPZ+AGT

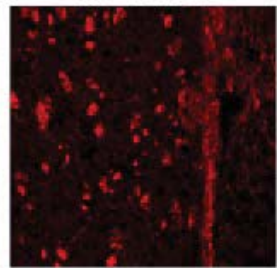

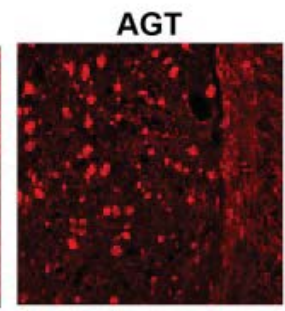

CPZ+AGT+RAPA

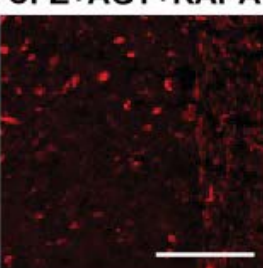

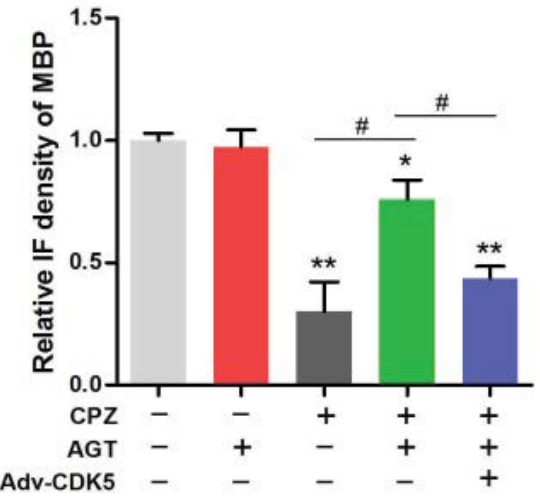

Figure 2: The effects of agomelatine and rapamycin on demyelination in CPZ-treated mice.

A. Western blotting analysis of LC3 I / II and MBP expression in ventrolateral striatum of mice.

B. Immunofluorescence staining and density analysis of MBP in ventrolateral striatum of mice. Scale bar: $150 \mu \mathrm{m}$. Data are expressed as mean \pm SEM $(n=10-$ 12 mice per group). ${ }^{*} P<0.05,{ }^{* *} P<0.01, "{ }^{*} P<0.05$.

pathology or antipsychotics in schizophrenia has been reported previously. And CDK5 also exerts regulations in cell-cycle progression, autophagy and survival. To explore whether CDK5 participated in CPZ-induced enhancement of autophagy, we measured the alteration of CDK5 expression and identified the effect of CDK5 modulation on the autophagy OLN93 cells. As was shown in Figure 4A, CPZ dose-dependently increased CDK5 expression $(P<0.05, P<0.01$, compared with the Control group). By using siRNAs targeted to CDK5 gene and CDK5 overexpression adenovirus, we found that siRNA-2 was most effective in suppressing CDK5 gene and protein expression out of all 3 siRNAs designed targeting CDK5 (data was not showed). Thus, siRNA-2 was used in the following experiments. In Figure 4B, Western blots showed overexpression of CDK5 in OLN93 cells resulted in LC3- II accumulation with or without $\mathrm{CPZ}$ treatment $(P<0.01$, compared with the Control group, respectively), whereas CDK5 knockdown exerted the converse effect by inhibiting the increase of LC3- II and CDK5 induced by CPZ $(P<0.05$, compared with the Control or CPZ group, respectively).

Accordingly, we observed the autophagic flux combined CPZtreatmentandCDK5 modulation. The results indicated CDK5 overexpression promoted while CDK5 knockdown antagonized the enhanced autophagic flux induced by CPZ. Compared with the Control group, silencing CDK5 had little effect on autophagic flux, but decreased the mRFP-LC3 dots. On the contrary, CDK5 overexpression promoted the autophagic process by decreasing the yellow dots tabbed autophagosomes and increasing the red dots tabbed autophagolysosomes $(P<0.01$, compared with the Control group). When concurrent of CPZ treatment, overexpression of CDK5 promoted the autophagy-inductive effect of 
A

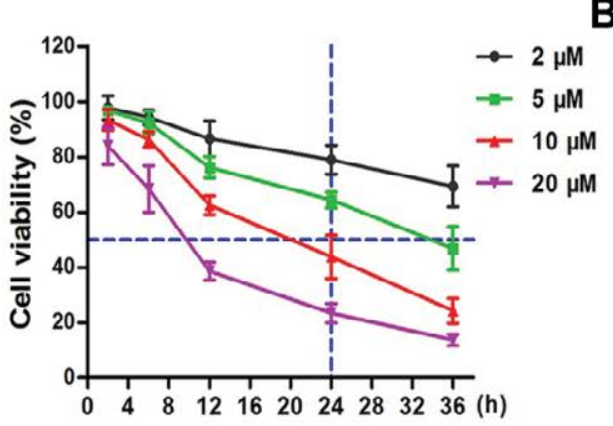

B
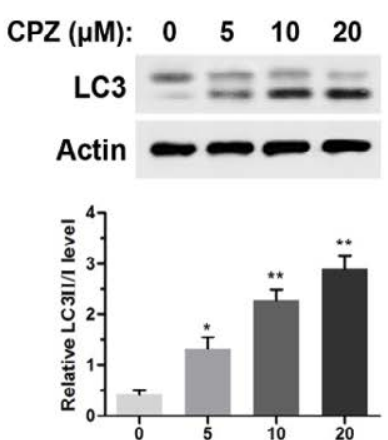

C $\operatorname{CPZ}(10 \mu \mathrm{M}): \quad 0 \quad 6 \quad 12 \quad 24 \mathrm{~h}$
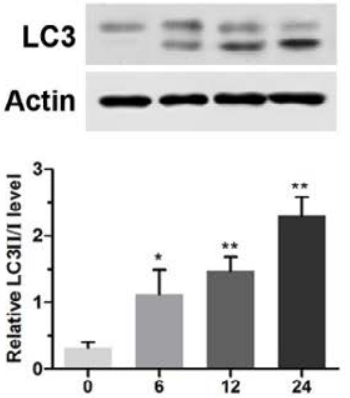

D

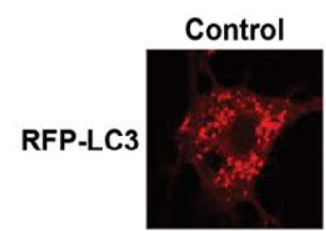

CPZ-5
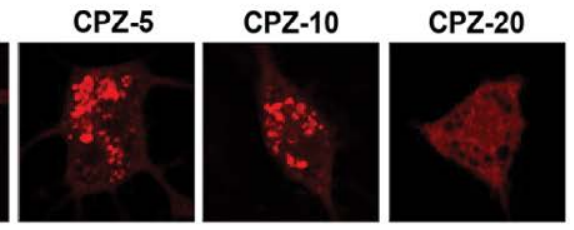

E
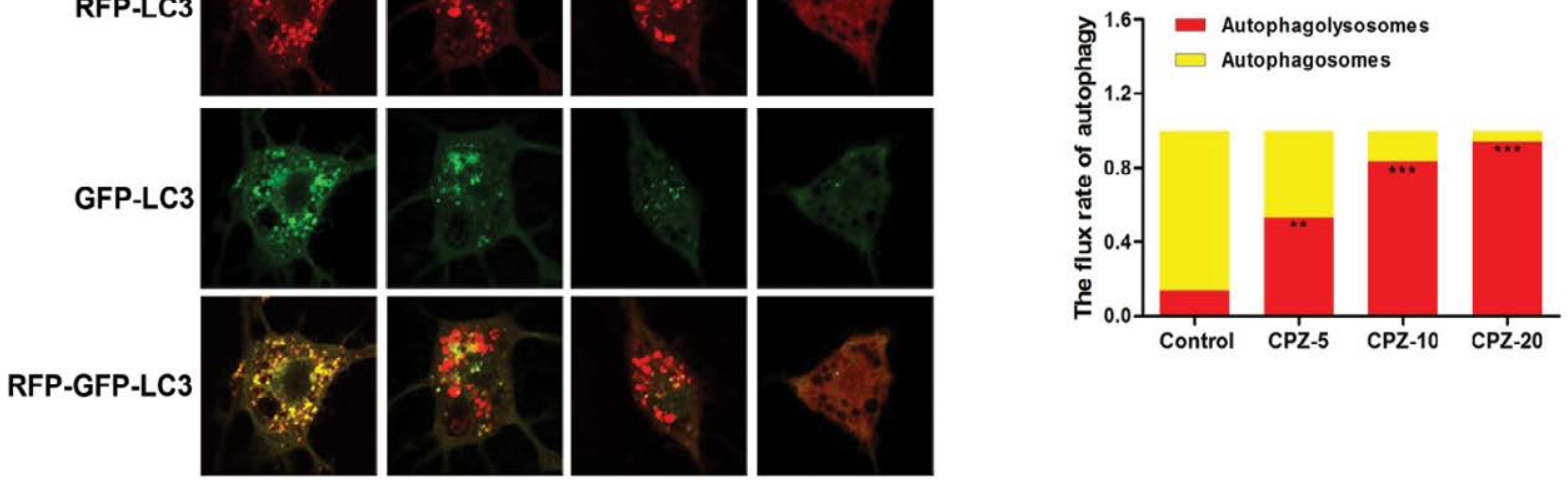

Figure 3: The effect of cuprizone on cell viability and autophagic process in OLN93 oligodendrocytes.

A. MTT assay of cell viability following different dose of cuprizone treatment.

B,C. Western blotting analysis of cuprizone dose- and time-dependently induced conversion of LC3. D. Representative images of the punctate RFP-GFPLC3 staining. E. The autophagic flux analysis by quantificating the percentage of autophagolysomes (red punctate / total punctate). Likewise, quantification of autophagosmes (yellow punctate) was measured as the percentage of (punctate yellow/total punctate signals) in the merged images. A minimum of 30 cells per group were counted. Data are expressed as mean $\pm \operatorname{SEM}(n=8)$. ${ }^{*} P<0.05,{ }^{* *} P<0.01,{ }^{* * *} P<0.001$.

$\mathrm{CPZ}(P<0.05$, compared with the CPZ group), while which was suppressed by siRNA of CDK5 $(P<0.05$, compared with the CPZ group). Together with the western blot results, these evidences indicated that the autophagy induced by CPZ was mediated by CDK5.

\section{- Agomelatine inhibited CPZ-induced excessive autophagy via suppression of CDK5}

Agomelatine is a melatonin analogue that serves as potent melatonin MT1/MT2 agonist, as well as partial 5-HT2C antagonist. Here, we used agomelatine for examining whether melatonergic treatment could reverse CPZ-induced aberrant autophagy in vitro. Western blots (Figure 5A) revealed the expressions of CDK5 and LC3II were significantly reduced by $20 \mu \mathrm{M}$ of agomelatine treatment $(P<0.01$, compared with the Control group, respectively). In addition, agomelatine $(20 \mu \mathrm{M})$ suppressed the elevation of CDK5 and LC3- II induced by CPZ $(P<0.05$, compared with the $\mathrm{CPZ}$ group, Figure $5 \mathrm{~B}$ ), which was blocked by CDK5 upregulation $(P<0.05$, compared with the $\mathrm{CPZ}+\mathrm{AGT}+\mathrm{Ad}-$ CDK5 group, Figure 5B). By determination of the autophagic flux (Figure 5C,D), we found that agomelatine alone had little effect on the autophagic flux. When being combined with CPZ treatment, additional agomelatine blocked the process of autophagy $(P<0.05$, compared with the $\mathrm{CPZ}$ group). Moreover, Compared with the $\mathrm{CPZ}+\mathrm{AGT}$ (agomelatine) group, the red-tabbed autophagolysosomes were increased and the yellowtabbed autophagosomes were decreased in the $\mathrm{CPZ}+\mathrm{AGT}+\mathrm{Adv}-\mathrm{CDK} 5$ group $(P<0.05)$, while which were weakened by CDK5-siRNA $(P<0.05)$. The above findings indicated agomelatine could resist the excessive autophagy induced by $\mathrm{CPZ}$, which might be attributed to CDK5. 
Potential Benefits of Melatonergic Treatment in Schizophrenia: Ameliorating Demyelination via Inhibiting CDK5-Mediated Hyper-Autophagy

A
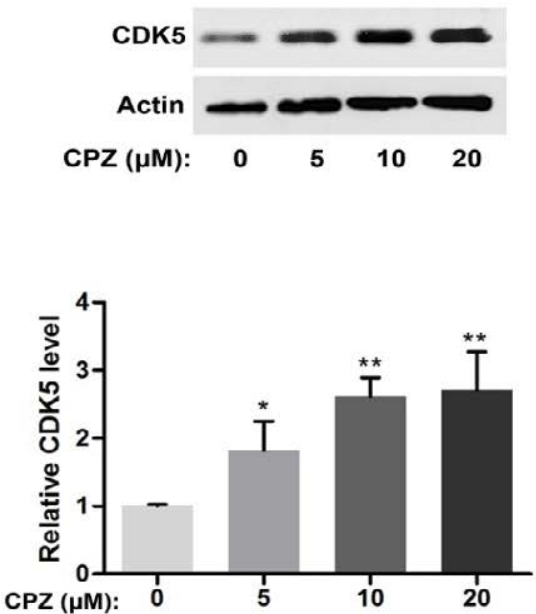

B
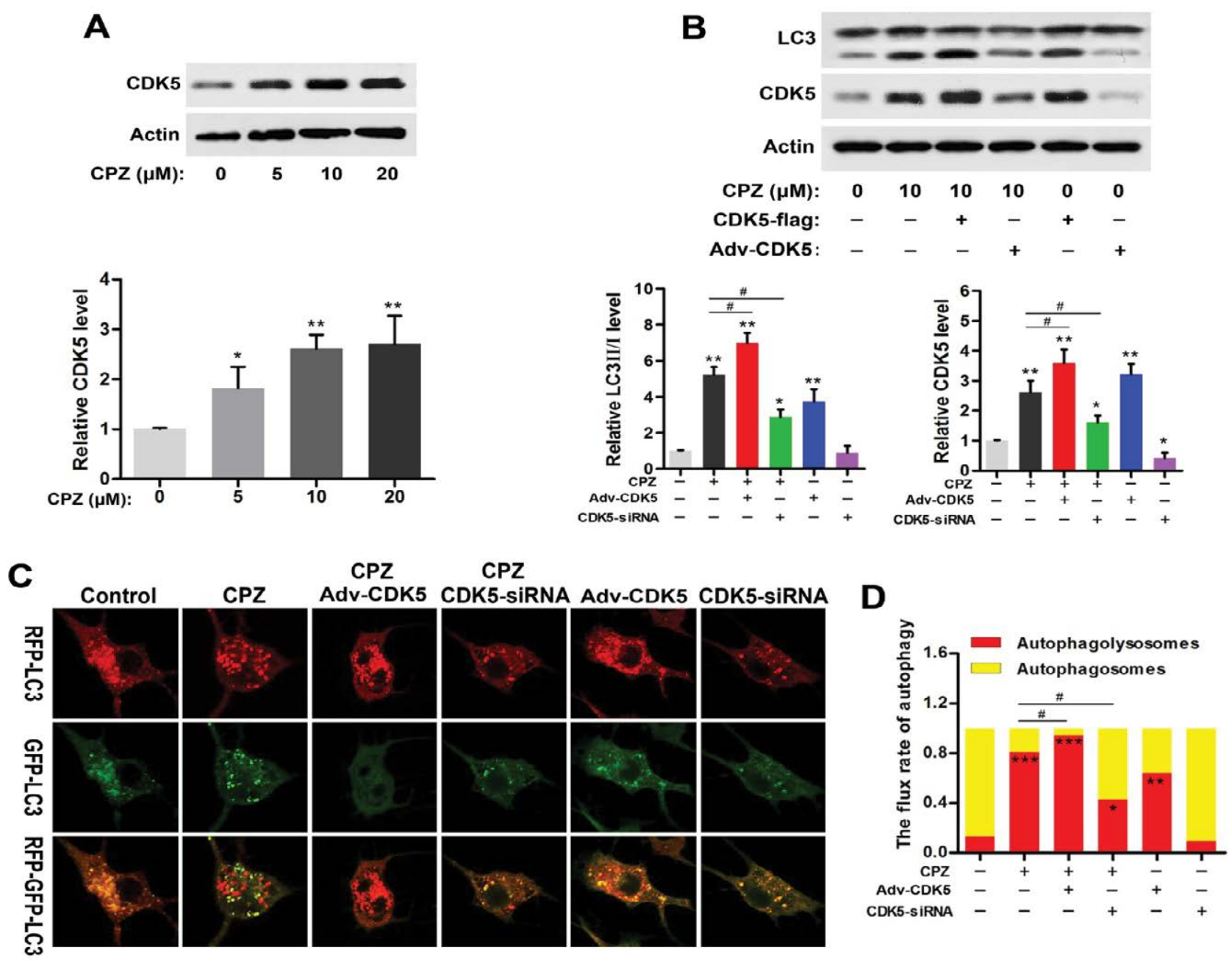

Figure 4: Cuprizone induced autophagy of oligodendrocyte in a CDK5-dependent manner.

A. Western blotting analysis of CDK5 expression induced by cuprizone in OLN93 cells. B. Western blotting analysis of LC3 and CDK5 levels following the indicated treatment in OLN93 cells. C,D. Representative images of the punctate RFP-GFP-LC3 staining and the autophagic flux analysis in OLN93 cells. A minimum of 30 cells per group were counted. Data are expressed as mean \pm SEM $(\mathrm{n}=8)$. ${ }^{*} P<0.05,{ }^{* *} P<0.01,{ }^{* * *} P<0.001,{ }^{*} P<0.05$.

\section{- Agomelatine inhibited activation of stress signaling and apoptosis in oligodendrocytes induced by CPZ via CDK5}

CPZ dose-dependently induced a phosphorylation of p38 and JNK $(P<0.05$, compared with the Control group, Figure 6A). Agomelatine inhibited the evaluations of p-p38 and p-JNK in CPZ-treated group (Figure 6B, $P<0.05$, compared with the CPZ group). AdvCDK5 weakened, while CDK5-siRNA enhanced the effect of agomelatine on the inhibition of p-p38 and p-JNK $(P<0.05$, compared with the CPZ+AGT group, respectively). These indicated that CDK5 was involved with the suppression of agomelatine on CPZ-induced p38 and JNK activation. Additionally, TUNEL staining results
(Figure 6C) illustrated that CPZ treatment led to an increase of cell apoptosis in vitro $(P<0.01$, compared with the Control group, Figure 6D). Agomelatine treatment alone did not affect the survival of oligodendrocytes. Fewer TUNEL positive cells were observed in the $\mathrm{CPZ}+\mathrm{AGT}$ group compared with the CPZ group $(P<0.05)$. The protective effects of agomelatine was abrogated by CDK 5 overexpression and enhanced by CDK5 knockdown $(P<0.05$, compared with the CPZ+AGT group), indicating that the antiapoptotic effect of agomelatine also might be dependent on the inhibition of CDK5.

\section{Discussion}

In spite of intensive investigation, the etiology and pathophysiology of schizophrenia 
A
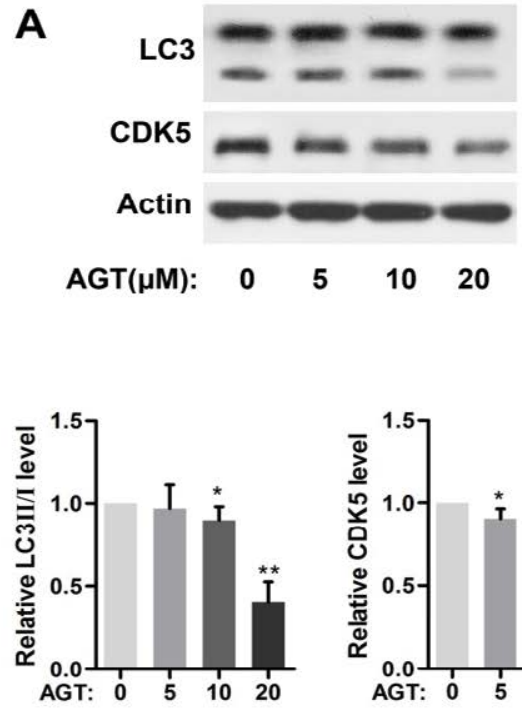

C
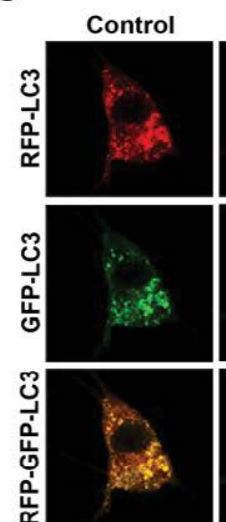
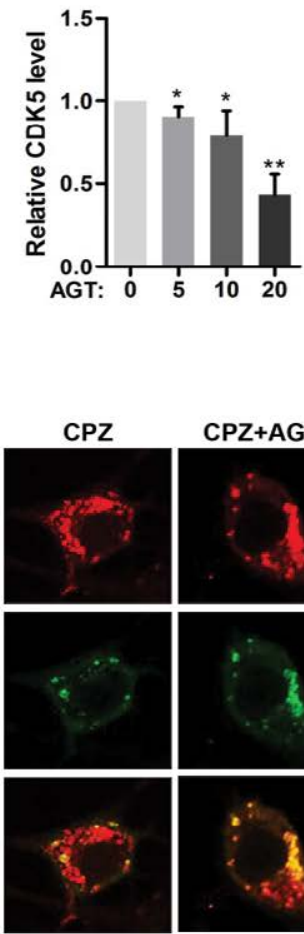

B
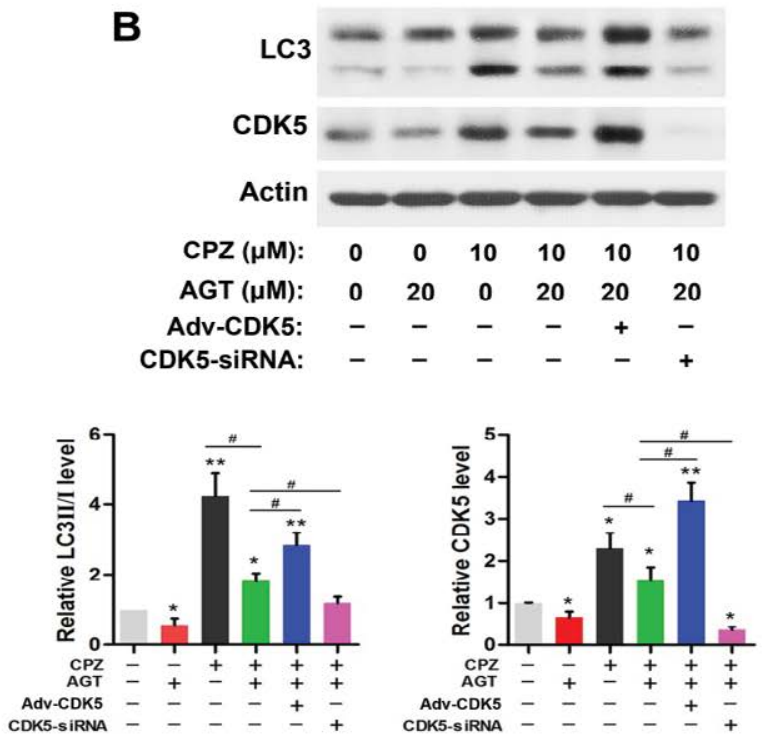

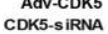
CDK5-siRNA
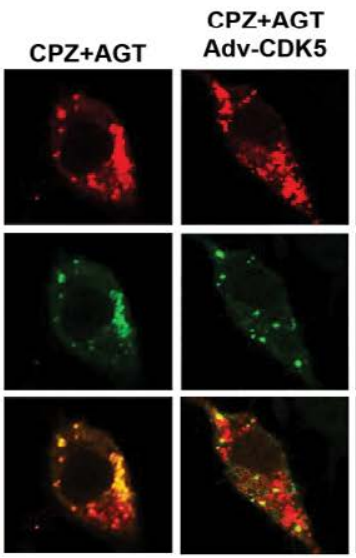

CPZ+AGT CDK5-SiRNA
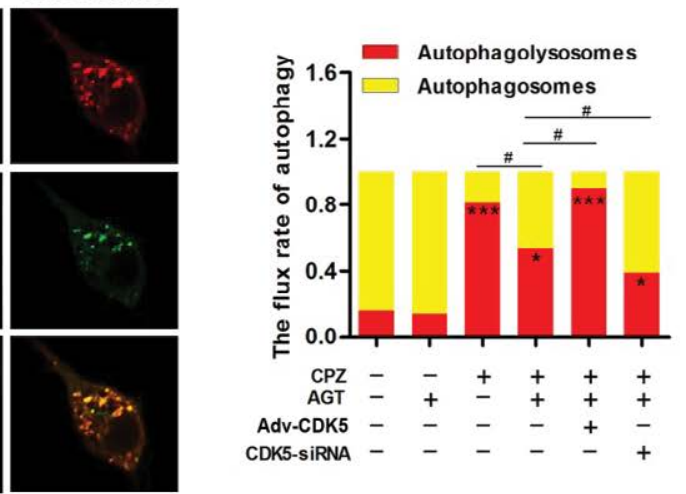

Figure 5: Agomelatine blocked CDK5-mediated autophagy induced by CPZ.

A. Western blotting analysis of LC3 and CDK5 expressions induced by cuprizone in OLN93 cells. B. Western blotting analysis of LC3 and CDK5 levels following the indicated treatment in OLN93 cells. C,D. Representative images of the punctate RFP-GFP-LC3 staining and the autophagic flux analysis in OLN93 cells. A minimum of 30 cells per group were counted. Data are expressed as mean $\pm \mathrm{SEM}(\mathrm{n}=8) .{ }^{*} P<0.05,{ }^{* * *} P<0.01,{ }^{*{ }^{* *}} P<0.001,{ }^{*} P<0.05$.

remains elusive. Although several hypotheses have been put it forward, no one could completely elaborate the disease process, as well as providing an effective therapeutic. The present study investigated the protection of melatonergic treatment in a CPZ-induced schizophrenia-like model, and further explored the underlying mechanism of demyelination and oligodendrocytic damages in vitro. We proposed a potential relationship between autophagy-demyelination and schizophrenia for the first time, and demonstrated CPZ-induced demyelination was involved with the excessive autophagy in oligodendrocytes, which finally leaded to the oligodendrocytic apoptosis. We also determined the critical roles of CDK5 for mediating autophagy in the toxical effect of $\mathrm{CPZ}$ and the protective effect of agomelatine. Our findings strongly suggested the potential benefits of melatinergic treatment with agomelatine against schizophrenia, and identified a novel mechanism of ameliorating demyelination by inhibiting CDK5-mediated autophagy in oligodendrocytes.

Among psychiatric disorders, schizophrenia is the most disabling and brings a large economic burden to both the family and the society. Although a group of specific genes were implicated in schizophrenia patients by screening differential expressed genes compared with the healthy population [6], lack of specificity still confused the clinical intervention. Melatonergic 
Potential Benefits of Melatonergic Treatment in Schizophrenia: Ameliorating Demyelination via Inhibiting CDK5-Mediated Hyper-Autophagy

A
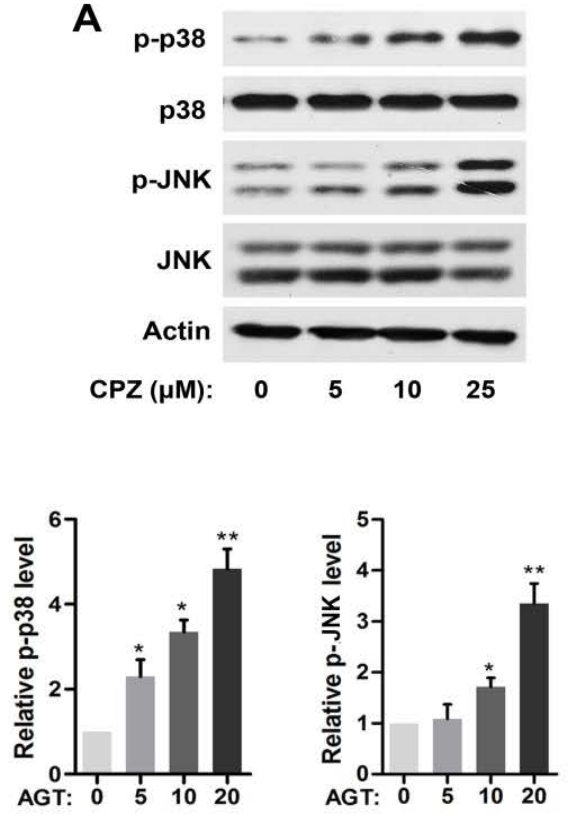

B
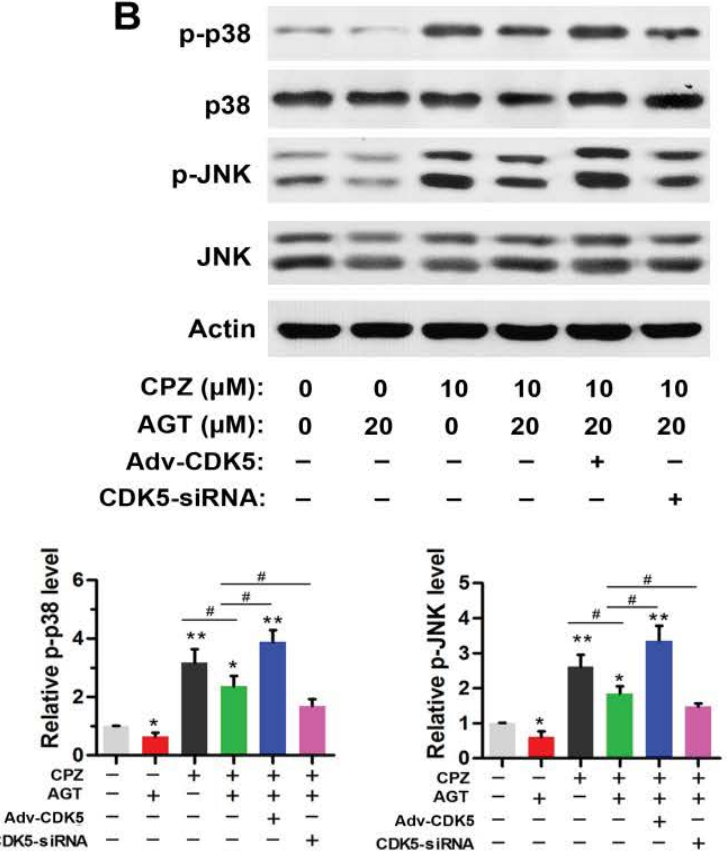

C
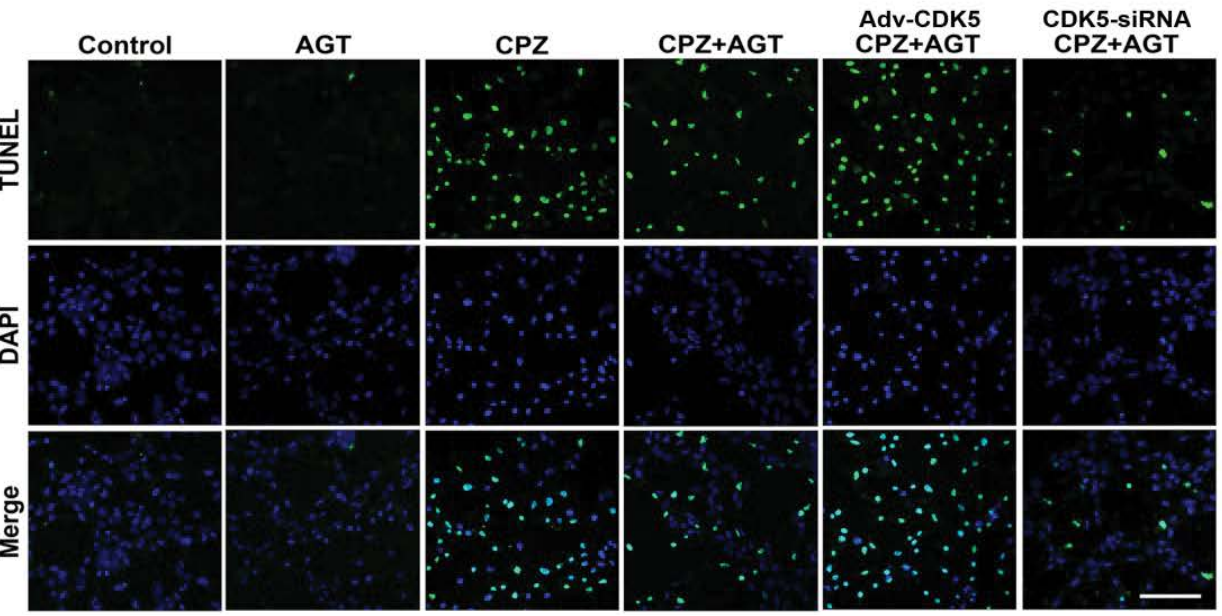

D

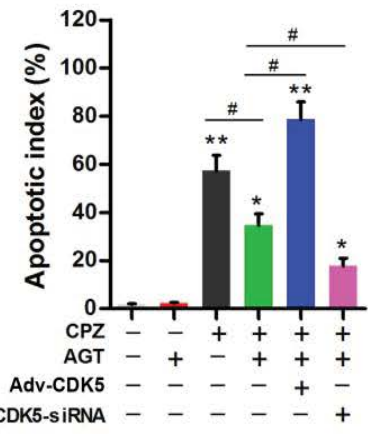

Figure 6: Agomelatine reversed the activation of p38 and JNK and attenuated cell death induced by CPZ via CDK5.

A. Western blotting analysis of p-p38, p38, p-JNK and JNK expressions induced by cuprizone in OLN93 cells. B. Western blotting analysis of p-p38, p38, p-JNK and JNK alterations following the indicated treatment in OLN93 cells. C,D. Apoptosis assay by TUNEL staining in OLN93 cells (C) and apoptotic index analysis (D). Scale bar: $40 \mu \mathrm{m}$. Data are expressed as mean \pm SEM $(n=8-12) .{ }^{*} P<0.05,{ }^{* *} P<0.01,{ }^{*} P<0.05$.

system has been indicated to be involved in schizophrenia. Either radiological investigation, endocrine monitoring or gene polymorphism $[14,15]$ analysis extremely suggested melatonin or MT agonist would possess potential for improving schizophrenia-like symptoms. Our present study confirmed that activating MT with agomelatine significantly alleviated the behavioral and cognitive deficits $[11,12]$, including negative symptoms, social interaction and working memory, which was in accordance with the previous clinical cases reports and experimental researches. Here, we partly identified the therapeutic effect of agomelatine on schizophrenia-like symptoms although the biological mechanism needed further investigation.

Clinical studies illustrated white matter abnormalities in schizophrenia patients. And animal models also demonstrated that demyelination caused behavioral manifestations reminiscent of schizophrenia. The period of myelination coincides with the same period 
when symptoms of most psychiatric disorders, especially schizophrenia begin to present. Oligodendrocyte abnormality and demyelination have recently been recognized as important pathophysiological basis for schizophrenia, alleviating which may be sufficient to reverse white matter pathologies of both patients and rodents models of schizophrenia [33]. Cuprizone is an oligodendrocyte specific toxin that is widely used to model the pathophysiology and phenotype of various demyelinating diseases including multiple sclerosis, depression and schizophrenia $[29,34,35]$. However, the underlying biological basis for the action of cuprizone is not fully elucidated. OLN93 cells are a group of cells derived from spontaneously transformed cells in primary rat brain glial cultures. Their antigenic properties, morphological features resemble 5 to 10 day-old (postnatal time) cultured rat brain oligodendrocytes, which make the OLN93 cells a useful model system to investigate the specific mechanisms regulating the proliferation and differentiation of oligodendrocytes in vitro [36].

Since autophagic flux is a dynamic process, it is insufficient to evaluate only autophagosome formation to detect the extent of autophagy. The mRFP-GFP-LC3 adenovirus is a useful tool to investigate the extent of autophagic flux, as the GFP is quenched in the acidic $\mathrm{pH}$ of the lysosomal compartment, while the mRFP continues to fluoresce, which can label the autophagic compartments both before and after fusion with lysosomes and analyze the autophagosome maturation process [37]. By using the mRFP-GFP-LC3 and determining the number of red dots that overlay green dots and appear yellow in merged images, the number of autophagosomes can be evaluated. The red dots that do not overlay green dots and appear red in merged images indicate autophagolysosome formation [38].

CDK5 is a proline directed serine / threonine kinase that has many important roles in the CNS, which is involved in neuron migration, neurite outgrowth and synaptogenesis. CDK5 participates in the regulation of autophagy to mediate neurotoxicity and neuronal cell death [39]. CDK5 expresses in oligodendrocytes and is important for oligodendrocyte maturation and myelination [40]. We found that in the OLN93 cells, CDK5 overproduction exacerbated while CDK5 inhibition by siRNA ameliorated cuprizone induced autophagy. Autophagy is essential for cell survival, however, extensive autophagy is commonly observed in dying cells, and acts as an executioner of cell death [41]. The autophagic cell death induced by cuprizone was blocked by agomelatine treatment confirmed the previous studies that cuprizone led to oligodendrocyte apoptosis [42]. CDK5 upregulation blocked while CDK5 knockdown enhanced the anti-apoptotic effect of agomelatine. In the meanwhile, Adv-CDK5 exacerbated and siRNA of CDK5 ameliorated CPZ-induced cell apoptosis, which indicated that agomelatine and cuprizone exerted their protective and injurious effects via regulation of CDK5 expression.

Agomelatine, a nonselective MT1 / MT2 melatonin receptor agonist and serotonergic 5-HT2C antagonist, possess many clinical applications. Previous studies demonstrated that agomelatine had anti-oxidative property which reduced oxidative stress, apoptosis, and inhibited cell death [43]. We provided evidence in the study that agomelatine ameliorated cuprizone induced apoptosis via inhibiting the CDK5mediated autophagy. In addition, the excessive autophagy was always accompanied by damaging cellular stress. Both p38 and JNK are important kinases for cellular stress and apoptosis. JNKs are important effectors of apoptosis in the brain both at transcriptional and translational levels. And activation of p38 MAPK is considered as part of the neuronal stress responses [44]. Previous studies reported that in the process of chronic stress in the endoplasmic reticulum (ER-stress), CDK5 activated the p38 and JNK pathway for apoptosis [45]. In our present study, we found that the phosphorylation of p38 and JNK was significantly increased by cuprizone, which was reversed by agomelatine treatment. Furthermore, CDK5 acted as a key regulator in mediating the activation of p38 and JNK. Beyond these, cuprizone induced activation of p38 and JNK may also result in apoptosis via inflammatory pathways [46]. Future in vivo experiments should be taken to investigate the biological and behavioral effects of agomelatine and CDK5 inhibition on CPZ induced rodent model of demyelination and remyelination to further confirm the efficiency of agomelatine in demyelinating diseases.

To summary, the present study, for the first time, linked autophagy-demyelination in schizophrenia, and suggested that melatinergic treatment with agomelatine possessed potential benefits against schizophrenia, and identified a novel mechanism of agomelatine ameliorating demyelination by inhibiting CDK5-mediated 
autophagy in oligodendrocytes. Our findings provided solid experimental evidences for the following extensive clinical trials of using melatinergic treatment in schizophrenia.
State Key Program of National Natural Science Foundation of China 81730035 (to WSX); the National Natural Science Foundation of China 81371240 (to WSX), 81500909 (to FDY).

\section{Sources of Funding}

\section{Disclosures}

This work was supported by grants from the None.

\section{References}

1. Global Health Observatory (GHO) data.

2. Mental health. Suicide data (2017).

3. Substance Abuse and Mental Health Services Administration, Results from the 2013 National Survey on Drug Use and Health: Mental Health Findings, NSDUH Series H-49, HHS Publication No. (SMA) 144887. Rockville, MD: Substance Abuse and Mental Health Services, 2014

4. Stoltenborgh M, Van ljzendoorn MH, Euser $\mathrm{EM}$, et al. A global perspective on child sexual abuse: Meta-analysis of prevalence around the world. Child maltreatment 16(2), 79-101(2011).

5. Fergusson DM, Mcleod GF, Horwood LJ. Childhood sexual abuse and adult developmental outcomes: Findings from a 30 -year longitudinal study in New Zealand. Child Abuse Neg/ 37(9), 664-674 (2013).

6. Berens $A E$, Jensen SKG, Nelson CA. Biological embedding of childhood adversity: from physiological mechanisms to clinical implications. BMCMe 15(1), (2017).

7. Afifi TO, MacMillan HL, Boyle M, et al. Child abuse and mental disorders in Canada. CMAJ 186(9), E324-E332 (2014).

8. Mills R, Scott J, Alati R, et al. Child maltreatment and adolescent mental health problems in a large birth cohort. Child Abuse \& Neglect 37(5), 292-302 (2013).

9. Stein MB, Campbell-Sills L, Ursano RJ, et al. Childhood maltreatment and lifetime suicidal behaviors among new Soldiers in the US Army: results from the Army Study to Assess Risk and Resilience in Servicemembers (Army STARRS). J Clin Psychiatry (2017).

10. Hoertel N, Franco S, Wall M, et al. Childhood Maltreatment and Risk of Suicide Attempt. J Clin Psychiatry 916-923 (2015).

11. Noll J. Sexual abuse of children-Unique in its effects on development? Child Abuse Neg 132(6), 603-605 (2008).

12. Fergusson DM, Boden JM, Horwood LJ. Exposure to childhood sexual and physical abuse and adjustment in early adulthood. Child Abuse Neg 32(6), 607-619 (2008).
13. Devries KM, Mak JYT, Child JC, et al. Childhood Sexual Abuse and Suicidal Behavior: A Meta-analysis. Pediatrics 133(5), (2014).

14. Suicide. Facts at a Glance (2015).

15. Grant RL. Converting an odds ratio to a range of plausible relative risks for better communication of research findings. $B M J$ 24(1), 348-f7450 (2014).

16. Barbosa LP, Quevedo L, da Silva GD, et al. Childhood trauma and suicide risk in a sample of young individuals aged 14-35 years in southern Brazil. Child abuse neg 38(7), 1191-1196 (2014)

17. Bernegger A, Kienesberger K, Carlberg L,et al. Influence of sex on suicidal phenotypes in affective disorder patients with traumatic childhood experiences. PLoS one 10(9), e0137763 (2015).

18. Brezo J, Paris J, Vitaro F, et al. Predicting suicide attempts in young adults with histories of childhood abuse. Br J Psychiatry 193(2), 134-139 (2008).

19. Briere J, Madni LA, Godbout N. Recent suicidality in the general population: multivariate association with childhood maltreatment and adult victimization. $J$ Interpers Violence 31(18), 3063-3079 (2016).

20. Brown J, Cohen P, Johnson JG, et al. Childhood abuse and neglect: specificity of effects on adolescent and young adult depression and suicidality. J Am Acad Child Adolesc Psychiatry 38(12), 1490-1496 (1999).

21. Carli V, Jovanović N, Podlešek A, et al. The role of impulsivity in self-mutilators, suicide ideators and suicide attempters-A study of 1265 male incarcerated individuals. J Affect Disord 123(1), 116-122 (2010).

22. Chowdhary N, Patel V. The effect of spousal violence on women's health: Findings from the Stree Arogya Shodh in Goa, India. Journal of postgraduate medicine 54(4), 306 (2008).

23. Clements-Nolle K, Wolden M, BargmannLosche J. Childhood trauma and risk for past and future suicide attempts among women in prison. Women's health issues 19(3), 185-92 (2009).

24. Cutajar MC, Mullen PE, Ogloff JR, et al. Suicide and fatal drug overdose in child sexual abuse victims: a historical cohort study. Med J Aust 192(4), 184-187 (2010).

25. De Bellis MD, Chrousos GP, Dorn LD, et al. Hypothalamic-pituitary-adrenal axis dysregulation in sexually abused girls. $J$ Clinical Endocrinology 78(2), 249-55 (1994).

26. Dinwiddie S, Heath AC, Dunne MP, et al. Early sexual abuse and lifetime psychopathology: a co-twin-control study. Psychol. Med 30(1), 41-52 (2000).

27. Enns MW, Cox BJ, Afifi TO, et al. Childhood adversities and risk for suicidal ideation and attempts: a longitudinal population-based study. Psychol. Med 36(12), 1769-1778 (2006).

28. Ernst C, Angst J, Földényi M. The Zurich Study. XVII. Sexual abuse in childhood. Frequency and relevance for adult morbidity data of a longitudinal epidemiological study. Eur. Arch. Psychiatry Clin. Neurosci 242(5), 293-300 (1993).

29. Ferraz L, Portella MJ, Vállez M, et al. Hostility and childhood sexual abuse as predictors of suicidal behaviour in Borderline Personality Disorder. Psychiatry res 210(3), 980-975 (2013).

30. Friestad C, Åse-Bente R, Kjelsberg E. Adverse childhood experiences among women prisoners: relationships to suicide attempts and drug abuse. Int J Soc Psychiatry 60(1), 40-46 (2014).

31. Güleç MY, Ýnanç L, Yanartap Ö, et al. Predictors of suicide in patients with conversion disorder. Compr Psychiatry 55(3), 457-462 (2014).

32. Hadland SE, Wood E, Dong $\mathrm{H}$, et al. Suicide attempts and childhood maltreatment among street youth: a prospective cohort study. Pediatrics 136(3), 440-449 (2015).

33. Harford TC, Yi HY, Grant BF. Associations between childhood abuse and interpersonal aggression and suicide attempt among US adults in a national study. Child abuse neg 38(8), 1389-1398 (2014).

34. Harvey EM, Rawson RA, Obert JL. History of sexual assault and the treatment of substance abuse disorders. J Psychoactive Drugs 26(4), 361-367 (1994).

35. Janiri D, Sani G, Danese E, et al. Childhood 
traumatic experiences of patients with bipolar disorder type I and type II. J affective disorders 175(1), 92-97 (2015).

36. Lopez CJ, Melhem N, Birmaher B, et al. Early childhood sexual abuse increases suicidal intent. World Psychiatry 12(2), 149-154 (2013).

37. Nelson EC, Heath AC, Madden PA, et al. Association between self-reported childhood sexual abuse and adverse psychosocial outcomes: results from a twin study. Arch Gen psychiatry 59(2), 139-145 (2002).

38. Pearce ME, Christian WM, Patterson K, et al. The Cedar Project: Historical trauma, sexual abuse and HIV risk among young Aboriginal people who use injection and non-injection drugs in two Canadian cities. Soc. Sci. Med 66(11), 2185-2194 (2008).

39. Pettigrew J, Burcham J. Effects of childhood sexual abuse in adult female psychiatric patients. Aust. N. Z. J. Psychiatry 31(2), 208213 (1997).

40. Plunkett A, O'Toole B, Swanston $\mathrm{H}$, et al. Suicide risk following child sexual abuse. Ambulatory Pediatrics 1(5), 262-266 (2001).

41. Rabinovitch SM, Kerr DC, Leve LD, et al. Suicidal behavior outcomes of childhood sexual abuse: Longitudinal study of adjudicated girls. Suicide Life Threat. Behav 45(4), 431-447 (2015).

42. Rimsza ME, Berg RA, Locke C. Sexual abuse: somatic and emotional reactions. Child abuse neg 12(2), 201-208 (1988).

43. Roy A. Reported childhood trauma and suicide attempts in schizophrenic patients. Life Threat. Behav 35(6), 690-693 (2005).

44. Roy A, Janal M. Gender in suicide attempt rates and childhood sexual abuse rates: is there an interaction? Suicide Life Threat. Behav 36(3), 329-335 (2006).

45. Sarchiapone M, Carli V, Cuomo C, et al. Childhood trauma and suicide attempts in patients with unipolar depression. Depress. Anxiety 24(4), 268-272 (2007).

46. Sarchiapone M, Jaussent I, Roy A, et al. Childhood trauma as a correlative factor of suicidal behavior-via aggression traits. Similar results in an Italian and in a French sample. Eur. Arch Psychiatry 24(1), 57-62 (2009).

47. Sfoggia A, Pacheco MA, Grassi-Oliveira R. History of childhood abuse and neglect and suicidal behavior at hospital admission. Crisis 29(3), 154-158 (2008).

48. Stewart JG, Kim JC, Esposito EC, et al. Predicting suicide attempts in depressed adolescents: Clarifying the role of disinhibition and childhood sexual abuse. J. Affect. Disord 187(1), 27-34 (2015).

49. Watson S, Gallagher P, Dougall D, et al. Childhood trauma in bipolar disorder. Aust. N. Z. J. Psychiatry 48(6), 564-570 (2014).

50. Yildirim F, Küçükgöncü S, Beştepe $E E$, et al. The relationship of childhood abuse and neglect with suicide attempts in an adult unipolar depression sample. Nöro Psikiyatri Arşivi 51(2),133 (2014).

51. Heim C, Nemeroff CB. The role of childhood trauma in the neurobiology of mood and anxiety disorders: preclinical and clinical studies. Bio. psychiatry 49(12), 1023-1039 (2001).

52. Heim C, Newport DJ, Heit S, et al. Pituitaryadrenal and autonomic responses to stress in women after sexual and physical abuse in childhood. JAMA 284(5), 592-597 (2000).
53. van Harmelen $A L$, van Tol MJ, van der Wee $\mathrm{NJ}$, et al. Reduced medial prefrontal cortex volume in adults reporting childhood emotional maltreatment. Bio. Psychiatry 68(9),832-838 (2010).

54. Etkin A, Wager TD. Functional neuroimaging of anxiety: a meta-analysis of emotional processing in PTSD, social anxiety disorder, and specific phobia. Am. J. Psychiatry 164(10), 1476-188 (2007).

55. Joiner Jr TE, Van Orden KA, Witte TK, et al. The interpersonal theory of suicide: Guidance for working with suicidal clients. Am. Psych. Association (2009).

56. Finkelhor D, Browne A. The traumatic impact of child sexual abuse: a conceptualization. Am. J orthopsychiatry 55(4), 530 (1985).

57. Cahill C, Llewelyn SP, Pearson C. Long-term effects of sexual abuse which occurred in childhood: A review. Br. J. Clin. Psychol 30(2), 117-130 (1991).

58. Lau M, Kristensen E. Outcome of systemic and analytic group psychotherapy for adult women with history of intrafamilial childhood sexual abuse: a randomized controlled study. Acta Psychiatr. Scand 116(2), 96-104 (2007).

59. Molnar BE, Berkman LF, Buka SL. Psychopathology, childhood sexual abuse and other childhood adversities: relative links to subsequent suicidal behaviour in the US. Psych medicine 31(6), 965-977 (2001)

60. Wyatt GE, Newcomb MD. Internal and external mediators of women's sexual abuse in childhood. J. Consult. Clin. Psychol 58(6),758 (1990). 